\title{
ON TRANSCENDENTAL MEROMORPHIC FUNCTIONS WHICH ARE GEOMETRICALLY FINITE
}

\author{
JIAN-HUA ZHENG
}

(Received 11 September 2000; revised 2 February 2001)

\author{
Communicated by P. C. Fenton
}

\begin{abstract}
In this paper we give the definition of a meromorphic function which is geometrically finite and investigate some properties of geometrically finite meromorphic functions and the Lebesgue measure of their Julia sets.
\end{abstract}

2000 Mathematics subject classification: primary 37F10, 37F50, 30D05.

Keywords and phrases: meromorphic function, geometrically finite, Julia set, Lebesgue measure.

\section{Introduction and results}

Let $f: \mathbb{C} \mapsto \hat{\mathbb{C}}$ be a transcendental meromorphic function, and $f^{n}, n \in \mathbb{N}$, denote the $n$th iterate of $f$. Then $f^{n}(z)$ is defined for all $z \in \mathbb{C}$ except for a countable set of the poles of $f, f^{2}, \ldots, f^{n-1}$. Define the Fatou set of $f$ by

$$
F(f)=\left\{z \in \mathbb{C} ;\left\{f^{n}\right\} \text { is defined and normal in some neighbourhood of } z\right\}
$$

and the Julia set of $f$ by $J(f)=\hat{\mathbb{C}} \backslash F(f)$. Put $J_{\infty}=\prod_{k=1}^{\infty} f^{-k}(\infty)$. Then $J_{\infty} \subset J(f)$, and all the $f^{n}(z)$ are analytic in $F(f)$ for each $n \in \mathbb{N}$. It is well known that $F(f)$ is open and completely invariant under $f$, that is, $z \in F(f)$ if and only if $f(z) \in F(f)$ and $J(f)$ also is completely invariant under $f$. Let $U$ be a connected component of $F(f)$. Then $f^{n}(U) \subseteq U_{n}$, where $U_{n}$ is a component of $F(f)$. If for $n \neq m, U_{n} \neq U_{m}$, then $U$ is called a wandering domain of $f$. For a wandering domain $U$, a basic result is that all the limit functions of $\left\{\left.f^{n}\right|_{U}\right\}$ are constants in $J(f) \cup\{\infty\}$. If for a smallest integer $p>0, f^{p}(U) \subseteq U$, then $U$ is said to be a periodic component

This work was supported by NSF of China and BRF of Tsinghua University.

(C) 2002 Australian Mathematical Society 0263-61 15/2002 \$A2.00+0.00 
of period $p$. If $U$ is a periodic component of period $p$ and there exists $a \in \partial U \cup\{\infty\}$ such that $f^{p n}(z) \rightarrow a$ in $U$ as $n \rightarrow \infty$, and $f^{P}(z)$ is not defined at $z=a$, then $U$ is called a Baker domain. Hence for a Baker domain $U$, all the limit functions of $\left\{\left.f^{n}\right|_{U}\right\}$ are constants in $J(f) \cup\{\infty\}$.

A point $z_{0}$ is called periodic if for some $n>0, f^{n}\left(z_{0}\right)=z_{0}$. In this case, the smallest $n$ with this property is called the period of $z_{0}$. A periodic point $z_{0}$ of period $n$ is called attracting, indifferent, or repelling according as $\left|\left(f^{n}\right)^{\prime}\left(z_{0}\right)\right|$ is less than, equal to, or greater than 1 . For an indifferent periodic point $z_{0}$ of period $n$, we have $\left(f^{n}\right)^{\prime}\left(z_{0}\right)=e^{2 \pi \alpha i}, 0 \leq \alpha<1$. When $\alpha$ is rational, we say that $z_{0}$ is rationally indifferent and when $\alpha$ is irrational, $z_{0}$ is irrationally indifferent and furthermore, in this case, $z_{0}$ is a Siegel point if $z_{0} \in F(f)$ or a Cremer point if $z_{0} \in J(f)$.

Denote by $\operatorname{sing}\left(f^{-1}\right)$ the set of singularities of the inverse function of $f$, that is, the set of critical and asymptotic values and limit points of these values. Define

$$
P(f)=\left\{w \in \mathbb{C}: \text { for some } n \in \mathbb{N}, f^{-n} \text { has a singularity at } w\right\}
$$

and

$$
S_{p}(f)=\bigcup_{k=0}^{p-1} f^{k}\left(\operatorname{sing}\left(f^{-1}\right) \backslash A_{k}\right),
$$

where $A_{k}=\left\{z: f^{k}\right.$ is not meromorphic at $\left.z\right\}, A_{0}=\emptyset$ and $A_{1}=\{\infty\}$. Then

$$
P(f)=\bigcup_{p=0}^{\infty} S_{p}(f) .
$$

DEFINITION. A meromorphic function $f(z)$ is called geometrically finite provided that

$$
\#\{J(f) \cap \overline{P(f)}\}<+\infty .
$$

When $f(z)$ is a rational function with degree at least 2, (1) implies that $f(z)$ has no Cremer points. This result is also true for the case when $f$ is of finite type, that is, the set $\operatorname{sing}\left(f^{-1}\right)$ is finite, since for a Cremer point $a$ of $f$, we have in $J(f)$ some singularity $c$ of $f^{-1}$ such that

$$
a \in \overline{O^{+}(c)} \backslash\left\{f^{n}(c)\right\}_{n=0}^{\infty}
$$

(see [9, Proposition 1.11]). Rational functions which are geometrically finite were investigated by several people. For example, it was proved in [10] (see [16, Chapter 5]) that the Julia set of such a rational function has Lebesgue measure zero. A meromorphic function which is geometrically finite and has no rationally indifferent periodic points is called subhyperbolic according to the case of rational functions, such rational functions have attracted much interest (see [16, Chapter 5]). 
In this paper, we discuss some basic properties and the Lebesgue measure of Julia sets of transcendental meromorphic functions which are geometrically finite. This case is obviously more complicated than that of rational functions. It is well known that the Julia set of $\lambda \tan z(0<\lambda<1)$ lies in the real axis (see [1, 4]) and the Julia set of $\sin z$ has positive Lebesgue measure (see [12]). These functions are all geometrically finite.

A point $a \in \mathbb{C}$ is said to be attracted by a set $X$ provided that $a$ is not (pre)periodic and all the limit points of $\left\{f^{n}(a)\right\}$ are in $X$. We denote by $C_{r}(f)$ the set of rationally indifferent periodic cycles of $f$, by $D(f)$ the set of points in $J(f)$ which are limit points of some sequence $\left\{f^{n_{k}}\left(b_{k}\right)\right\}$, where $b_{k} \in F(f) \cap \operatorname{sing}\left(f^{-1}\right)$ such that $b_{k} \rightarrow b \in J(f)$ and $n_{k} \rightarrow \infty(k \rightarrow \infty)$, and by $L(f)$ the set of all the limit functions of $\left.f^{n}\right|_{U}$, where $U$ is a wandering or Baker domain containing at least one element of $\operatorname{sing}\left(f^{-1}\right)$.

THEOREM 1. A meromorphic function $f(z)$ is geometrically finite if and only if all of the following statements hold:

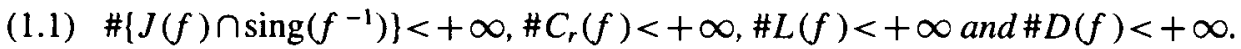
(1.2) For each $a \in J(f) \cap \operatorname{sing}\left(f^{-1}\right)$ either a is (pre)periodic or $f^{n}(a)=\infty$ for some $n>0$.

(1.3) Each $b \in F(f) \cap \operatorname{sing}\left(f^{-1}\right)$ is attracted by either (super)attracting or rationally indifferent periodic cycles or for some $n>0, f^{n}(b)$ is periodic or there exists a subsequence $\left\{n_{k}\right\}$ of positive integers süch that $f^{n_{k}}(b) \rightarrow \infty(k \rightarrow \infty)$.

For the case of entire functions we can deduce the following.

THEOREM 2. An entire function $f(z)$ is geometrically finite if and only if all of the following statements hold:

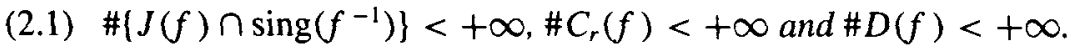

(2.2) For each $a \in J(f) \cap \operatorname{sing}\left(f^{-1}\right)$, a is (pre)periodic.

(2.3) Each $b \in F(f) \cap \operatorname{sing} f^{-1}$ is attracted by either (super)attracting or rationally indifferent periodic cycles, or for some $n>0, f^{n}(b)$ is periodic or $f^{n}(b) \rightarrow \infty$, $n \rightarrow \infty$.

We denote by $B_{p}$ the family of meromorphic functions with bounded $S_{p}(f)$. We write $B_{1}$ as $B$. The dynamics of the functions in Class $B$ have been investigated by many authors, see, for example, $[3,7,18]$. For an integer $p>0$, put $I_{p}(f):=\{z \in$ $\mathbb{C}: f^{n p}(z) \rightarrow \infty$ as $n \rightarrow \infty$ and $f^{n p}(z) \neq \infty$. We write $I_{1}(f)$ as $I(f)$. Eremenko [6] proved that if $f$ is entire, then $I(f) \neq \emptyset$ and $J(f)=\partial I(f)$, this was extended by Dominguez [5] to the case of meromorphic functions. It was proved by Zheng [18] that for $f \in B, l(f) \subset J(f)$ so that $J(f)=\overline{l(f)}$. Below we shall discuss the 
Lebesgue measure of $I_{p}(f)$ and $J(f)$. For $a \in \mathbb{C}, r>0$ and $R>0$, define

$$
D(a, r):=\{z ;|z-a|<r\} \text { and } D_{R}:=\mathbb{C} \backslash D(0, R) .
$$

The following concept is important. A subset $E$ of $\mathbb{C}$ is called thin at infinity provided that there exist $r>0$ and $R>0$ such that for all $a \in E \cap D_{R}$, we have

$$
\operatorname{density}(E, D(a, r))=\frac{\operatorname{mes}(E \cap D(a, r))}{\operatorname{mes}(D(a, r))}<1-\varepsilon,
$$

where $\varepsilon>0$ is independent of $a$. The definition is a little different from that given in [12].

THEOREM 3. Let $f$ be in Class $B_{p}$. If $I_{p}(f)$ is thin at infinity, then mes $I_{p}(f)=0$. If, in additon, $f$ is geometrically finite and $J(f) \cap \overline{P(f)} \cap J_{\infty}=\emptyset$, then mes $J(f)=0$.

REMARK 1. McMullen [12] proved that $\operatorname{mes}(I(\sin z))>0$. It is clear that $\sin z$ is of finite type and geometrically finite. Hence ' $I_{p}(f)$ is thin at infinity' cannot be left out in Theorem 3. However, Eremenko and Lyubich [7] proved under different assumptions that for an entire $f \in B$, mes $I(f)=0$.

THEOREM 4. Let $f$ be a geometrically finite meromorphic function. Assume that there exists $R>0$ such that dist $\left(I(f) \cap J(f) \cap D_{R}, \overline{P(f)}\right)>0$. If $I(f) \cap J(f)$ is thin at infinity and $J(f) \cap \overline{P(f)} \cap J_{\infty}=\emptyset$, then mes $J(f)=0$.

THEOREM 5. Let $f$ be a geometrically finite meromorphic function. Assume that there exists $R>0$ such that $\operatorname{dist}\left(J(f) \cap D_{R}, \overline{P(f)}\right)>0$. If $J(f)$ is thin at infinity, then mes $J(f)=0$.

REMARK 2. Theorem 4 is an extension of [ 8 , Theorem 3$]$. Theorem 5 was proved by McMullen [12] in the case when $f$ is entire with compact $\overline{P(f)}$ and $J(f) \cap \overline{P(f)}=\emptyset$ and by Stallard [15] in the case when $f$ is entire with dist $(J(f), \overline{P(f)})>0$.

It is not easy in general to determine whether the Julia set of a meromorphic function is thin at infinity. It is obviously easier to decide that $I(f) \cap J(f)$ is thin at infinity, and so it is sometimes more convenient to use Theorem 4 to show that mes $J(f)=0$. We discuss this further in the final section.

By the method of Stallard [15] and McMullen [12], we can deduce the following result, which is used in the proofs of Theorems 3-5 and which is of independent interest.

THEOREM 6. Let $f$ be a meromorphic function with $J(f) \neq \hat{\mathbb{C}}$ and let $E$ be a completely invariant subset of $J(f)$ under $f$. For $a \in E \backslash J_{\infty}$, if there exists a subsequence $\left\{m_{k}\right\}$ of positive integers satisfying $\operatorname{dist}\left(f^{m_{k}}(a), \overline{P(f)}\right) \geq \delta>0$, $k=1,2, \ldots$, then the following statements hold: 
(6.1) $\left|\left(f^{m_{k}}\right)^{\prime}(a)\right| \rightarrow \infty, k \rightarrow \infty$.

(6.2) Either a is not a Lebesgue density point of $E$ or $f^{m_{k}}(a) \rightarrow \infty, k \rightarrow \infty$, and in this case, if, in addition, there exists $R>0$ such that $\sigma:=\operatorname{dist}\left(E \cap D_{R}, \overline{P(f)}\right)>0$, then $\operatorname{dist}\left(f^{m_{k}}(a), \overline{P(f)}\right) \rightarrow \infty$.

\section{Proofs of Theorems 1 and 2}

In order to prove Theorem 1 and Theorem 2 in this paper, we need the following results.

THEOREM 7. Let $f$ be a transcendental meromorphic function. If $a \notin J_{\infty}$, then a cannot be attracted by any repelling or irrationally indifferent cycles. If, in addition, $a$ is not in any parabolic domain, then a cannot be attracted by any rationally indifferent cycles.

Theorem 7 was proved by Perez-Marco [11] in the case of irrationally indifferent cycles and by using the Petal Theorem of Fatou in the case of rationally indifferent cycles. It is obvious that Theorem 7 holds for the case of repelling cycles.

THEOREM 8. Let $f$ be a transcendental meromorphic function. If $U$ is a wandering domain of $f$, then all the limit functions of $\left\{\left.f^{n}\right|_{U}\right\}$ lie in $(P(f))^{\prime} \cap J(f)$. If $U$ is a Baker domain of period $p$ of $f$, then all the limit functions of $\left\{\left.f^{n}\right|_{U}\right\}$ lie in $\left(S_{p}(f)\right)^{\prime} \cap J(f)$.

Theorem 8 was proved by Zheng [17]. The following lemma will be often used in the proofs of our Theorem 1, Theorem 2 and Theorem 3 (see [8]).

LEMMA 1. Let $f$ be a transcendental meromorphic function and let $a \notin J_{\infty}$ and assume that a is not (pre)periodic. Assume that the set $S$ of limit points of $\left\{f^{n}(a)\right\}$ in the sphere $\hat{\mathbb{C}}$ is finite. Then one of the following statements holds:

(I) if $\left\{f^{n}(a)\right\}$ has a finite limit point which is not in $J_{\infty}$, a is attracted by a periodic cycle;

(II) there exists a subsequence $\left\{n_{k}\right\}$ of positive integers such that $f^{n_{k}}(a) \rightarrow \infty$ $(k \rightarrow \infty)$, and in this case, if, in addition, $(S \backslash\{\infty\}) \cap J_{\infty}=\emptyset$, then $f^{n}(a) \rightarrow \infty$ $(n \rightarrow \infty)$.

PROOF. Let $\left\{a_{1}, a_{2}, \ldots, a_{m}\right\}$ be the set of all finite limit points of $\left\{f^{n}(a)\right\}$. We take positive numbers $d$ and $M$ such that $D\left(a_{j}, d\right)(j=1,2, \ldots, m)$ are mutually disjoint and lie inside $D(0, M)$. Then there exists a positive integer $n_{0}$ such that for $n>n_{0}, f^{n}(a) \in \bigcup_{j=1}^{m} D\left(a_{j}, d\right) \cup\{|z|>M\}$. We can take $r>0$ and an integer $n_{1}>n_{0}>0$ such that when $n>n_{1}$, we have $f^{n}(a) \in \bigcup_{j=1}^{m} D\left(a_{j}, r\right) \cup\{|z|>M\}$ 
and $f\left(D\left(a_{j}, r\right)\right) \subset D\left(f\left(a_{j}\right), d\right)$, where we replace $D\left(f\left(a_{j}\right), d\right)$ with $\{|z|>2 M\}$ for $f\left(a_{j}\right)=\infty$. Now we assume, without loss of generality, that there is a subsequence $f^{n_{k}}(a) \rightarrow a_{1}(k \rightarrow \infty)$. If $a_{1} \notin J_{\infty}$, then for $k>n_{1}$, we have $f^{n_{k}}(a) \in D\left(a_{1}, r\right)$, and hence $f^{n_{k}+1}(a) \in D\left(f\left(a_{1}\right), d\right)$, and by noting that $f\left(a_{1}\right)$ is also a limit point of $\left\{f^{n}(a)\right\}$, we have $f^{n_{k}+1}(a) \in D\left(f\left(a_{1}\right), r\right)$. Thus we deduce that for $n>n_{k}$, $f^{n}(a) \in \bigcup_{j=1}^{m} D\left(f^{j}\left(a_{1}\right), r\right)$, and $a_{1}$ is (pre)periodic and furthermore from $f^{n_{k}}(a) \rightarrow$ $a_{1}(k \rightarrow \infty)$ it is easy to see that $a_{1}$ is periodic. Therefore $a$ is attracted by a periodic cycle. On the other hand, if for some $p>0, f^{p}\left(a_{1}\right)=\infty$, then $f^{n_{k}+p}(a) \rightarrow \infty$ $(k \rightarrow \infty)$.

If $f^{n}(a) \nrightarrow \infty(n \rightarrow \infty)$ and $(S \backslash\{\infty\}) \cap J_{\infty}=\emptyset$, the same argument as above implies that $a$ is attracted by a periodic cycle.

PROOF OF THEOREM 1. We prove that (1.1), (1.2) and (1.3) in Theorem 1 hold provided that $f$ is geometrically finite. It is obvious that (1.1) holds. For $a \in$ $J(f) \cap \overline{P(f)}=A$ (say), it is clear that $\left\{f^{n}(a)\right\} \subset A$, and from (1) we have that for some $n>0, f^{n}(a)$ is periodic or $\infty$. Consequently, (1.2) follows.

Now we prove (1.3). Obviously, $F(f)$ has no Siegel disks and Herman rings, since it is well known that their boundaries belong to $J(f) \cap \overline{P(f)}$. Let $U$ be the component of $F(f)$ containing $b$. We only need to consider the case when $U$ is wandering. From Theorem 8, we have that all the limit functions of $\left\{f^{n}(b)\right\}$ are in $J(f) \cap(P(f))^{\prime}$ and from Theorem 7, $b$ cannot be attracted to a periodic cycle in $J(f)$. Lemma 1 implies (1.3).

Below we prove that $f$ is geometrically finite provided that (1.1), (1.2) and (1.3) hold. Since $F(f)$ is completely invariant under $f, O^{+}(F(f))$ cannot meet $J(f)$, and so we have

$$
\begin{aligned}
J(f) \cap P(f) & =O^{+}\left(J(f) \cap \operatorname{sing}\left(f^{-1}\right)\right) \cup\left(O^{+}\left(F(f) \cap \operatorname{sing}\left(f^{-1}\right)\right) \cap J(f)\right) \\
& =O^{+}\left(J(f) \cap \operatorname{sing}\left(f^{-1}\right)\right) .
\end{aligned}
$$

Since \# $\left(J(f) \cap \operatorname{sing}\left(f^{-1}\right)\right)<+\infty$, it follows from (1.2) that we have

$$
p:=\# O^{+}\left(J(f) \cap \operatorname{sing}\left(f^{-1}\right)\right)<+\infty \text { and }(J(f) \cap P(f))^{\prime}=\emptyset .
$$

Note that

$$
\begin{aligned}
J(f) \cap(P(f))^{\prime} & =J(f) \cap(F(f) \cap P(f))^{\prime} \\
& =\left[O^{+}\left(F(f) \cap \operatorname{sing}\left(f^{-1}\right)\right)\right]^{\prime} \cap J(f) .
\end{aligned}
$$

For $a \in\left[O^{+}\left(F(f) \cap \operatorname{sing}\left(f^{-1}\right)\right)\right]^{\prime} \cap J(f)$, there exists a sequence $b_{k} \in F(f) \cap$ $\operatorname{sing}\left(f^{-1}\right)$ and $n_{k} \in \mathbb{N}$ such that $f^{n_{k}}\left(b_{k}\right) \rightarrow a(k \rightarrow \infty)$. If there is a subsequence $b_{k_{j}}=b \in F(f) \cap \operatorname{sing}\left(f^{-1}\right)$, then $a \in C_{r}(f) \cup L(f)$. If $b_{k} \neq b_{k^{\prime}}\left(k \neq k^{\prime}\right)$ and $\left\{b_{k}\right\}$ has a limit point in $F(f)$, then it follows from (1.3) that $a \in C_{r}(f) \cup L(f)$. If $b_{k} \neq b_{k^{\prime}}$ $\left(k \neq k^{\prime}\right)$ and $b_{k} \rightarrow b \in J(f) \cap \operatorname{sing}\left(f^{-1}\right)$ and $n_{k}=p$, then $f^{n_{k}}\left(b_{k}\right)=f^{p}\left(b_{k}\right) \rightarrow$ 
$f^{p}(b)=a(k \rightarrow \infty)$ so that $a \in O^{+}\left(J(f) \cap \sin f^{-1}\right)$. Under the remaining case, $a \in D(f)$. Therefore,

$$
\left[O^{+}\left(F(f) \cap \operatorname{sing} f^{-1}\right)\right]^{\prime} \cap J(f) \subset C_{r}(f) \cup L(f) \cup D(f) \cup O^{+}\left(J(f) \cap \operatorname{sing} f^{-1}\right) .
$$

Thus we deduce

$$
\#(J(f) \cap \overline{P(f)}) \leq 2 p+\# C_{r}(f)+\# L(f)+\# D(f)<+\infty,
$$

so that $f$ is geometrically finite.

PROOF OF THEOREM 2. We prove that (2.1), (2.2) and (2.3) hold provided that $f$ is geometrically finite. By Theorem 1 we only need to prove (2.3). Let $U$ be the component of $F(f)$ containing $b$. Obviously, we only need to consider the case when $U$ is wandering. From Theorem 7, $\left\{f^{n}(b)\right\}$ cannot be attracted by any periodic cycles in $J(f)$. Since all the limit points of $\left\{f^{n}(b)\right\}$ are in $J(f) \cap P(f)^{\prime}$ and $f$ is geometrically finite, Lemma 1 implies $f^{n}(b) \rightarrow \infty(n \rightarrow \infty)$.

Now we prove that $f$ is geometrically finite using (2.1), (2.2) and (2.3). For $a \in L(f) \backslash\{\infty\}$, we have a component $U$ of $F(f)$ and $b \in U \cap \operatorname{sing}\left(f^{-1}\right)$ such that $a$ is a limit point of $\left\{f^{n}(b)\right\}$. Then $U$ is wandering. By (2.3) we derive a contradiction. Thus $L(f) \backslash\{\infty\}=\emptyset$. Then the same argument as in the proof of Theorem 1 implies the desired result.

\section{Proofs of Theorem 3 and Theorem 4}

In the proofs of Theorem 3 and Theorem 4, we need the following lemma, which is essentially due to [7].

LEMMA 2. Assume that $f \in B_{p}$ and $0 \notin \bigcup_{k=1}^{\infty} f^{-k}(\infty)$. Then there exists a positive constant d such that for all $z \in \mathbb{C} \backslash\{0\}$ at which $f^{p}$ is analytic, we have

$$
\left|\left(f^{p}\right)^{\prime}(z)\right| \geq \frac{\left|f^{p}(z)\right|}{4|z|} \log \frac{\left|f^{p}(z)\right|}{d} .
$$

A proof of Lemma 2 was given in [14] for ' $16 \pi$ ' instead of ' 4 ' in (3) by the logarithmic change of variable in a neighbourhood of infinity, as in [7, Lemma 1]. We prove (3) using the hyperbolic metric principle (see [19]).

PROOF. Take a positive number $d$ such that

$$
\operatorname{sing}\left(f^{-p}\right) \subset D(0, d) \text { and }\left|f^{p}(0)\right|<d .
$$


Let $z_{0}$ be an analytic point of $f^{p}$ such that $\left|f^{p}\left(z_{0}\right)\right|>d$. There exists a domain $U$ such that $z_{0} \in U$ and $f^{p}: U \rightarrow D_{d}$ is a covering map. By the hyperbolic metric principle, we have

$$
\lambda_{U}\left(z_{0}\right)=\lambda_{D_{d}}\left(f^{p}\left(z_{0}\right)\right)\left|\left(f^{p}\right)^{\prime}\left(z_{0}\right)\right|=\frac{\left|\left(f^{p}\right)^{\prime}\left(z_{0}\right)\right|}{\left|f^{p}\left(z_{0}\right)\right| \log \left(\left|f^{p}\left(z_{0}\right)\right| / d\right)},
$$

where $\lambda_{U}$ and $\lambda_{D_{d}}$ are the hyperbolic densities of $U$ and $D_{d}$ respectively. Let $U^{*}$ be the component of $f^{-p}\left(D_{d} \cup\{\infty\}\right)$ containing $U$. From [7, page 993] or [14, Lemma 2.1], $U^{*}$ is simply connected and $0 \notin U^{*}$. Thus we have

$$
\lambda_{U}\left(z_{0}\right)>\lambda_{U^{*}}\left(z_{0}\right) \geq \frac{1}{4 \operatorname{dist}\left(z_{0}, \partial U\right)} \geq \frac{1}{4\left|z_{0}\right|} .
$$

Combining the above inequality with (4) implies (3).

The following is an immediate consequence of Lemma2.

COROLLARY. Let $f$ be in $B_{p}$. For $z \in I_{p}(f)$, we have

$$
\frac{\left|\left(f^{n p}\right)^{\prime}(z)\right|}{\left|f^{n p}(z)\right|} \rightarrow \infty, \quad \text { as } n \rightarrow \infty \text {. }
$$

Therefore, $f^{p}$ is expanding on $I_{p}(f)$.

PROof. Indeed, by (3) we have

$$
\left|\left(f^{n p}\right)^{\prime}(z)\right| \geq \frac{\left|f^{n p}(z)\right|}{|z|} \prod_{k=0}^{n-1} \frac{1}{4} \log \frac{\left|f^{k p}(z)\right|}{d}, z \in I_{p}(f) .
$$

Then (5) follows.

PROOF OF THEOREM 3. Assume, without loss of generality, that $0 \notin \bigcup_{k=1}^{\infty} f^{-k}(\infty)$, otherwise we consider the function $g(z)=f(z+c)-c$, where $c \notin \bigcup_{k=1}^{\infty} f^{-k}(\infty)$, then $0 \notin \bigcup_{k=1}^{\infty} g^{-k}(\infty)$, and note that mes $I_{p}(f)=\operatorname{mes} I_{p}(g)$ and mes $J(f)=\operatorname{mes} J(g)$. Since $I_{p}(f)$ is thin at infinity and $f \in B_{p}$, we can find $R, r, \varepsilon>0$ such that $R \geq 2 r$,

$$
\operatorname{sing}\left(f^{-p}\right) \cap D_{R / 2}=\emptyset
$$

and for all $z \in I_{p}(f) \cap D_{2 R}$, we have

$$
\operatorname{density}\left(I_{p}(f), D(z, r)\right)<1-\varepsilon
$$

and $\log (|z| / d) \geq 32$, where $d$ is the constant in Lemma 2. Given $a \in I_{p}(f) \cap D_{4 R}$, put $a_{m}:=f^{m p}(a)$. Since $f^{n p}(a)$ is a Lebsegue density point of $I_{p}(f)$ if and only if $a$ is, 
by noting that $I_{p}(f)$ is completely invariant under $f^{p}$, we have $a_{m} \rightarrow \infty(m \rightarrow \infty)$, and we can assume $\left|a_{m}\right| \geq 4 R, m=0,1,2, \ldots$, and $D\left(a_{m}, 2 R\right) \subset D_{R}$. Then there exists the analytic branch $f_{m}^{-p}$ of $f^{-p}$ that is univalent in the disk $D\left(a_{m}, 2 R\right)$ and that maps $a_{m}$ to $a_{m-1}$. It follows from Koebe's Distortion Theorem that

$$
f_{m}^{-p}\left(D\left(a_{m}, R\right)\right) \subset D\left(a_{m-1}, 4 R\left|\left(f_{m}^{-p}\right)^{\prime}\left(a_{m}\right)\right|\right) .
$$

From Lemma 2 we have

$$
\begin{aligned}
\left|a_{m-1}\right|-8 R\left|\left(f_{m}^{-p}\right)^{\prime}\left(a_{m}\right)\right| & =\left|a_{m-1}\right|-\frac{8 R}{\left|\left(f^{p}\right)^{\prime}\left(a_{m-1}\right)\right|} \\
& \geq\left|a_{m-1}\right|-\frac{32 R\left|a_{m-1}\right|}{\left|a_{m}\right|}\left[\log \frac{\left|a_{m}\right|}{d}\right]^{-1} \\
& \geq\left|a_{m-1}\right|-\frac{R\left|a_{m-1}\right|}{\left|a_{m}\right|}>R,
\end{aligned}
$$

and hence

$$
D\left(a_{m-1}, 8 R\left|\left(f_{m}^{-p}\right)^{\prime}\left(a_{m}\right)\right|\right) \cap \operatorname{sing}\left(f^{-p}\right)=\emptyset .
$$

By the same argument as above, we have

$$
f_{m-1}^{-p}\left(D\left(a_{m-1}, 4 R\left|\left(f_{m}^{-p}\right)^{\prime}\left(a_{m}\right)\right|\right)\right) \subset D\left(a_{m-2}, 4 R\left|\left(f_{m}^{-p}\right)^{\prime}\left(a_{m}\right)\right| 4\left|\left(f_{m-1}^{-p}\right)^{\prime}\left(a_{m-1}\right)\right|\right)
$$

and by induction, we have

$$
f^{-m p}\left(D\left(a_{m}, R\right)\right) \subset D\left(a, R \prod_{k=1}^{m} 4\left|\left(f_{k}^{-p}\right)^{\prime}\left(a_{k}\right)\right|\right),
$$

where $f^{-m p}=f_{1}^{-p} \circ f_{2}^{-p} \circ \cdots \circ f_{m}^{-p}$ is univalent in $D\left(a_{m}, 2 r\right)$ and hence Koebe's Distortion Theorem implies that $f^{-m p}$ has distortion $K=9$ in $D\left(a_{m}, r\right)$. Using Koebe's Distortion Theorem again we have that

$$
D\left(a, s_{m} / 16\right) \subset D_{m}:=f^{-m p}\left(D\left(a_{m}, r\right)\right) \subset D\left(a, s_{m}\right),
$$

where $s_{m}$ is the radius of the smallest disk centered at $a$ containing $D_{m}$, and furthermore, we have

$$
\begin{aligned}
0<s_{m} & \leq R \prod_{k=1}^{m} 4\left|\left(f_{k}^{-p}\right)^{\prime}\left(a_{k}\right)\right|=R \prod_{k=1}^{m} \frac{4}{\left|\left(f^{p}\right)^{\prime}\left(a_{k-1}\right)\right|} \\
& \leq \frac{R|a|}{\left|a_{m}\right|} \prod_{k=0}^{m-1} 16\left[\log \frac{\left|a_{k}\right|}{d}\right]^{-1} \rightarrow 0, \quad m \rightarrow \infty .
\end{aligned}
$$


From (6) and noting that $I_{p}(f)$ is completely invariant under $f^{p}$, we deduce

$$
\operatorname{density}\left(I_{p}(f), D_{m}\right)<1-\varepsilon / 81 .
$$

Thus $a$ is not a point of Lebesgue density of $I_{p}(f)$, and mes $I_{p}(f)=0$.

Now we prove the latter part of Theorem 3. Since $I(f) \subset I_{p}(f)$, we have mes $I(f)=0$. For any $a \in J(f) \backslash\left(I(f) \cup J_{\infty}\right),\left\{f^{n}(a)\right\}$ has at least one finite limit point. If all the limit points of $\left\{f^{n}(a)\right\}$ are in $J(f) \cap \overline{P(f)}$, then from Lemma 1 and since $J(f) \cap \overline{P(f)} \cap J_{\infty}=\emptyset$, we have that $a$ is attracted by a periodic cycles. However, this contradicts to Theorem 7 . Therefore there exists a subsequence $\left\{m_{k}\right\}$ of positive integers such that $b_{k}:=f^{m_{k}}(a) \rightarrow b \in J(f)$, but $b \notin J(f) \cap \overline{P(f)} \cup\{\infty\}$. Then we have that for some $\delta>0$, dist $\left(b_{k}, \overline{P(f)}\right) \geq \delta>0$. From (6.2) of Theorem6 it follows that $a$ is not a point of Lebesgue density of $J(f)$, and hence not of $J(f) \backslash\left(I(f) \cup J_{\infty}\right)$. We derive mes $\left(J(f) \backslash\left(I(f) \cup J_{\infty}\right)\right)=0$, and since $J_{\infty}$ is countable, it follows that mes $J(f)=0$.

PROOF OF THEOREM 4. Analysing the proof of the latter part of Theorem 3, we only need to prove mes $(I(f) \cap J(f))=0$. Suppose that $I(f) \cap J(f)$ has a Lebesgue density point $a$. Since $a_{n}:=f^{n}(a) \rightarrow \infty(n \rightarrow \infty)$, we can assume that $\left|a_{n}\right|>$ $R, n=1,2, \ldots$, so that $\operatorname{dist}\left(\left\{a_{n}\right\}, \overline{P(f)}\right)>0$. Then by applying Theorem 6 to $I(f) \cap J(f)$ we have $\left(f^{n}\right)^{\prime}(a) \rightarrow \infty$ and $\operatorname{dist}\left(a_{n}, \overline{P(f)}\right) \rightarrow \infty(n \rightarrow \infty)$. Since $I(f) \cap J(f)$ is thin at infinity, there exist $r, \varepsilon>0$ such that

$$
\operatorname{density}\left(I(f) \cap J(f), D\left(a_{n}, r\right)\right)<1-\varepsilon .
$$

Put $r_{n}:=(1 / 2) \operatorname{dist}\left(a_{n}, \overline{P(f)}\right)$. We can assume that $r_{n}>r, n=1,2, \ldots$ There exists an analytic branch $g_{n}$ of $f^{-n}$ univalent in $D\left(a_{n}, 2 r_{n}\right)$ carrying $f^{n}(a)$ back to $a$. Koebe's Distortion Theorem implies that

$$
D\left(a, d_{n}\right) \subset U_{n}:=g_{n}\left(D\left(a_{n}, r\right)\right) \subset D\left(a, 16 d_{n}\right),
$$

where $d_{n}:=(r / 4)\left|g_{n}^{\prime}\left(a_{n}\right)\right|=r /\left(4\left|\left(f^{n}\right)^{\prime}(a)\right|\right) \rightarrow 0, n \rightarrow \infty$. Thus from (7) it follows that $a$ is not a Lebesgue density point of $I(f) \cap J(f)$. We derive a contradiction, from which Theorem 4 follows.

PROOF OF THEOREM 5. For a given point $a \in J(f) \backslash\left(\overline{P(f)} \cup J_{\infty}\right)$, we want to prove that $a$ is not a Lebesgue density point of $J(f)$. To this end suppose that $a$ is. We consider two cases.

(i) Assume that there exists a subsequence $\left\{m_{k}\right\}$ of positive integers such that $\left\{f^{m_{k}}(a)\right\} \subset D_{R}$, then by Theorem 6 , we have $b_{k}:=f^{m_{k}}(a) \rightarrow \infty$ and $\operatorname{dist}\left(b_{k}, \overline{P(f)}\right) \rightarrow$ $\infty(k \rightarrow \infty)$, otherwise $a$ is not a Lebesgue density point of $J(f)$. By the same argument as in the proof of Theorem 4 we can derive a contradiction. 
(ii) Assume that $\left|f^{n}(a)\right| \leq R, n=1,2, \ldots$. Since $f$ is geometrically finite, from Lemma 1 and Theorem 7 it follows that $\left\{f^{n}(a)\right\}$ has a limit point not in $\overline{P(f)} \cap J(f) \cup$ $\{\infty\}$. Therefore for some constant $\delta>0$ and a subsequence $\left\{n_{k}\right\}$ of positive integers, $\operatorname{dist}\left(f^{n_{k}}(a), \overline{P(f)}\right) \geq \delta$. By (6.2) of Theorem 6, we deduce that $a$ is not a Lebesgue density point of $J(f)$.

\section{Proof of Theorem 6}

PROOF OF THEOREM 6. We prove (6.1). Since $D\left(f^{m_{k}}(a), \delta\right) \cap \overline{P(f)}=\emptyset$, there exists the univalent branch $g_{k}$ of $f^{-m_{k}}$ in $D\left(f^{m_{k}}(a), \delta\right)$ carrying $f^{m_{k}}(a)$ back to $a$. By Koebe's Quarter Theorem,

$$
g_{k}\left(D\left(f^{m_{k}}(a), \delta\right)\right) \supset D\left(a,(\delta / 4)\left|g_{k}^{\prime}\left(f^{m_{k}}(a)\right)\right|\right),
$$

and hence

$$
f^{m_{k}}\left(D\left(a,(\delta / 4)\left|g_{k}^{\prime}\left(f^{m_{k}}(a)\right)\right|\right)\right) \subset D\left(f^{m_{k}}(a), \delta\right) .
$$

For any fixed neighbourhood $V$ of $a \in J(f), f^{n}(V)$ contains a disk with radius $2 \delta$, when $n$ is sufficiently large. This implies that $(\delta / 4)\left|g_{k}^{\prime}\left(f^{m_{k}}(a)\right)\right| \rightarrow 0$, that is, $\left(f^{m_{k}}\right)^{\prime}(a) \rightarrow \infty, k \rightarrow \infty$.

Now we prove (6.2). To this end, we assume that $a$ is a Lebesgue density point of $J(f)$. Put $b_{k}:=f^{m_{k}}(a)$ and $C_{k}:=g_{k}\left(D\left(b_{k}, \delta / 2\right)\right)$.

Suppose that $\left\{b_{k}\right\}$ has a bounded subsequence, and assume, without loss of generality, that $b_{k} \rightarrow b \in J(f), k \rightarrow \infty$. Koebe's Distortion Theorem implies that

$$
D\left(a, \rho_{k}\right) \subset C_{k} \subset D\left(a, 16 \rho_{k}\right),
$$

where $\rho_{k}:=\left|g_{k}^{\prime}\left(b_{k}\right)\right| \delta / 8=\delta /\left[8\left|\left(f^{m_{k}}\right)^{\prime}(a)\right|\right] \rightarrow 0$, as $k \rightarrow \infty$, where we employed (6.1).

From (8) we have

so that

$$
\lim _{k \rightarrow \infty} \frac{\operatorname{mes}\left(C_{k} \backslash J(f)\right)}{\operatorname{mes}\left(C_{k}\right)} \leq(16)^{2} \lim _{k \rightarrow \infty} \frac{\operatorname{mes}\left(D\left(a, 16 \rho_{k}\right) \backslash J(f)\right)}{\operatorname{mes}\left(D\left(a, 16 \rho_{k}\right)\right)}=0,
$$

$$
\lim _{k \rightarrow \infty} \frac{\operatorname{mes}\left(D\left(b_{k}, \delta / 2\right) \cap J(f)\right)}{\operatorname{mes}\left(D\left(b_{k}, \delta / 2\right)\right)}=1,
$$

and consequently, $\operatorname{mes}(D(b, \delta / 2) \cap J(f))=\operatorname{mes}(D(b, \delta / 2))$. Thus $D(b, \delta / 2) \subset$ $J(f)$, and $J(f)=\hat{\mathbb{C}}$. This is a contradiction. Therefore $b_{k} \rightarrow \infty$ as $k \rightarrow \infty$.

Now we prove the latter part of (6.2). Suppose conversely that for some $M>0$, $r_{k}:=\operatorname{dist}\left(b_{k}, \overline{P(f)}\right)<M$. Since $b_{k} \rightarrow \infty, k \rightarrow \infty$, we can assume that $\left|b_{k}\right|>$ $R+M, k=1,2, \ldots$. We can find $z_{k} \in \overline{P(f)}$ such that $\left|z_{k}-b_{k}\right|=\operatorname{dist}\left(b_{k}, \overline{P(f)}\right)$ 
and hence $\left|z_{k}\right|>R, k=1,2, \ldots$ It is clear that $D\left(z_{k}, \sigma\right) \cap E=\emptyset$, otherwise $\operatorname{dist}\left(E \cap D_{R}, \overline{P(f)}\right)<\sigma$, and then mes $\left(D\left(z_{k}, \sigma\right) \cap D_{k}\right)>0$, where $D_{k}:=D\left(b_{k}, s_{k}\right)$, $s_{k}=r_{k}-\sigma / 4$. It follows that

$$
\operatorname{density}\left(E, D_{k}\right)<1-\varepsilon(M, \sigma)<1,
$$

where $\varepsilon(M, \sigma)$ only depends on $M$ and $\sigma$. Put $U_{k}:=g_{k}\left(D_{k}\right)$. Koebe's Distortion Theorem implies that

$$
D\left(a, d_{k}\right) \subset U_{k} \subset D\left(a, 16 d_{k}\right),
$$

and $0<d_{k}:=s_{k}\left|g_{k}^{\prime}\left(b_{k}\right)\right| / 4 \leq M /\left(4\left|\left(f^{m_{k}}\right)^{\prime}(a)\right|\right) \rightarrow 0, k \rightarrow \infty$. Then from (9) it follows that $a$ is not a Lebesgue density point of $E$. We derive a contradiction.

\section{Conclusion}

From the previous discussion we have seen that, to some extent, whether the Julia set of a meromorphic function has Lebesgue measure zero depends on whether $I(f) \cap J(f)$ is thin at infinity. When $f(z)$ is iñ Class B, Eremenko and Lyubich [7] gave simple criteria for $I(f)$ being thin at infinity, for example, $f$ is an entire function of finite order and its inverse $f^{-1}$ has a logarithmic singularity. Hence mes $I\left(e^{2}\right)=0$, but it is well known that $J\left(e^{z}\right)=\hat{\mathbb{C}}$ (see [13]). Since $e_{n}(0) \rightarrow \infty(n \rightarrow \infty)$, where $e_{n}(z)$ is the $n$th iterate of $e^{z}, e^{z}$ is not geometrically finite. This is very interesting. From their method, we can deduce that if $f$ is an entire function of finite order and its inverse $f^{-1}$ has a logarithmic singularity, then $I\left(f\left(e^{z}\right)\right)$ is thin at infinity. The following is an example illustrating Theorem 3 .

EXAMPLE 1. Julia sets of $z e^{z}$ and $\exp \left(z-e^{z-1}\right)$ have Lebesgue measure zero.

PROOF. It is easy to see that $z e^{z}$ is of finite type and 0 is only one asymptotic value of $z e^{z}$ and a logarithmic singularity of the inverse. From the result of Eremenko and Lyubich [7], we have mes $\left(I\left(z e^{z}\right)\right)=0$. It is easy to see that 0 is a rationally indifferent fixed point of $z e^{z}$ and $z=-1$ is the only critical point of $z e^{z}$ so that -1 is attracted to 0 under iterates of $z e^{z}$, and $-1 \in F\left(z e^{z}\right)$. Thus $z e^{z}$ is geometrically finite. Theorem 3 implies $\operatorname{mes}\left(J\left(z e^{z}\right)\right)=0$.

Let $h(z)=\exp \left(z-e^{z-1}\right)$. A simple calculation implies that $\operatorname{sing}\left(h^{-1}\right)=\{0,1\}$ and 1 is a superattracting fixed point of $h(z)$. We prove that $h^{n}(0) \rightarrow 1(n \rightarrow \infty)$. For $0 \leq x<1, h^{\prime}(x)>0$ and $h(x)<h(1)=1$. Note that

$$
(h(x)-x)^{\prime}=\left(1-e^{x-1}\right) h(x)-1<0,
$$


and so $h(x)-x>h(1)-1=0$, that is, $h(x)>x$ so that $h([0,1]) \subset(0,1]$ and further $h^{n}(0) \rightarrow 1(n \rightarrow \infty)$. Thus $h$ is geometrically finite. We can write $h(z)=$ $\left(w e^{-w / e}\right) \circ e^{z}$, and hence $I(h)$ is thin at infinity. Theorem 3 implies mes $(I(h))=$ $\operatorname{mes}(J(h))=0$.

The next example illustrates Theorem 5 .

EXAMPLE 2. The Julia set of $f(z)=z+4+e^{-z}+\varepsilon /(z-1), \varepsilon=0.02$ has Lebesgue measure zero and $H:=\{z ; \operatorname{Re} z>0$ and $|z-1|>0.1\} \subset I(f)$.

PROOF. For all $z \in H$, we have

$$
\operatorname{Re} f(z)=\operatorname{Re} z+4+\operatorname{Re}\left(e^{-z}+\varepsilon /(z-1)\right)>4-1-0.2>2,
$$

so that $f(H) \subset H$, and $H \subset F(f)$. Note that

$$
f^{\prime}(z)=1-e^{-z}-\varepsilon /(z-1)^{2} .
$$

When $|z-1|<0.1$, we have

$$
\left|f^{\prime}(z)\right| \geq \frac{\varepsilon}{|z-1|^{2}}-1-\left|e^{-z}\right|>2-1-\exp (-1+0.1)>0,
$$

and therefore all the critical points of $f(z)$ lie in $D:=\{z ;|z-1|>0.1\}$. When $z \in D$ and $f^{\prime}(z)=0$, it follows from (10) that $e^{-z}=1-\varepsilon /(z-1)^{2}$ and so we have

$$
\left|e^{-z}\right| \leq 1+2=3 \text { and } \operatorname{Re} z \geq-\log 3
$$

so that

$$
\begin{aligned}
\operatorname{Re} f(z) & \geq \operatorname{Re} z+5-\operatorname{Re} \frac{\varepsilon}{(z-1)^{2}}+\operatorname{Re} \frac{\varepsilon}{z-1} \\
& \geq-\log 3+5-2-0.2=-\log 3+2.8>1.3
\end{aligned}
$$

Therefore, $\operatorname{sing}\left(f^{-1}\right) \subset H$, and further $\overline{P(f)} \subset H$ and $\operatorname{dist}(\overline{P(f)}, J(f))>0.2$. Hence $f$ is geometrically finite.

Below we prove that $J(f)$ is thin at infinity. Obviously there exists a negative number $x_{0}$ such that for $x \leq x_{0}$, we have $d(x):=x+4-\varepsilon+\sqrt{2} e^{-x} / 2>4$. Put $H_{k}:=\{-\pi / 4+2 k \pi<\operatorname{Im} z<\pi / 4+2 k \pi\} \cap\left\{\operatorname{Re} z<x_{0}\right\}, k=0, \pm 1, \pm 2, \ldots$ For $z \in H_{k}$, we have

$$
\operatorname{Re} f(z)=\operatorname{Re} z+4+\operatorname{Re}(\varepsilon /(z-1))+e^{-\operatorname{Re} z} \cos (\operatorname{Im} z) \geq d(\operatorname{Re} z)>4 .
$$

We deduce that $f\left(H_{k}\right) \subset H$ and $H_{k} \subset F(f)$. This implies that $J(f)$ is thin at infinity. By Theorem 5, we have that mes $J(f)=0$. It is obvious that $H \subset I(f)$. 
EXAMPLE 3. Let $g(z)=\lambda \sin z-\varepsilon /(z-\pi)$, where $\lambda$ and $\varepsilon$ are both non-zero constants. Then mes $(I(g))>0$ and $\operatorname{mes}(J(g))>0$.

ProOF. We first note that $g(z)$ has no asymptotic values. We now consider the critical values of $g(z)$. For $z \in \mathbb{C}$ such that

$$
g^{\prime}(z)=\lambda \cdot \cos z+\frac{\varepsilon}{(z-\pi)^{2}}=0
$$

we have

$$
\begin{aligned}
|g(z)| & \leq|\lambda||\sin z|+\frac{|\varepsilon|}{|z-\pi|} \leq|\lambda| \sqrt{1+\left|\cos ^{2} z\right|}+\frac{|\varepsilon|}{|z-\pi|} \\
& \leq|\lambda| \sqrt{1+\frac{|\varepsilon|^{2}}{|\lambda|^{2}|z-\pi|^{4}}}+\frac{|\varepsilon|}{|z-\pi|} .
\end{aligned}
$$

Since the sequence of the zeros of $g^{\prime}(z)$ tends to infinity, from (11) we deduce $\operatorname{sing}\left(g^{-1}\right)$ is bounded and infinite, that is, $g(z)$ is in Class B.

By the same argument as in [12], we can prove that mes $(I(g))>0$. This is because for sufficiently large $h>0,\left|g^{\prime}(z)\right|=O\left(e^{h}\right)$ and $\left|g^{\prime \prime}(z) / g^{\prime}(z)\right| \approx 1$ in $\{z ;|\operatorname{Im}(z)|>h\}$, that is, $g$ is expanding by a rate of exponentiation and the nonlinearity is near 1 . By [18, Theorem 2], we have $I(g) \subset J(g)$ and hence $\operatorname{mes}(J(f))>0$.

It was proved in [5] that for sufficiently small $\varepsilon$ and $0<\lambda<1, F(g)$ consists of one attracting basin and contains all the singularities of $g^{-1}$. It is easy to see that $g(z)$ is geometrically finite and $\operatorname{dist}(\overline{P(g)}, J(g))>0$.

We conclude the section with the following result.

Let $f(z)$ be an entire function having a completely invariant component. If $\operatorname{sing}\left(f^{-1}\right)$ consists of critical values and logarithmic singularities and their limit points, and $\operatorname{sing}\left(f^{-1}\right) \cap J(f)$ is finite, then $f$ is geometrically finite.

The result follows from [7, Lemma 11], which asserts that a completely invariant component contains all the critical values and logarithmic singularities.

\section{References}

[1] I. N. Baker, J. Kotus and Y. Lu, 'Iterates of meromorphic functions I', Ergodic Theory Dynam. Systems 11 (1991), 241-248.

[2] A. F. Beardon and Ch. Pommerenke, 'The Poincaré metric of plane domains', J. London Math. Soc. (2) 18 (1978), 475-483.

[3] W. Bergweiler, 'Iteration of meromorphic functions', Bull. Amer. Math. Soc. (New Series) 29 (1993), 151-188.

[4] R. L. Devaney and L. Keen, 'Dynamics of meromorphic maps with polynomial Schwarzian derivative', Ann. Sci. Ecole Norm. Sup. 22 (1989), 55-81. 
[5] P. Dominguez, 'Dynamics of transcendental meromorphic functions', Ann. Acad. Sci. Fenn. Ser A.I. Math. 23 (1998), 225-250.

[6] A. E. Eremenko, 'On the iteration of entire functions', in: Dynamical systems and ergodic theory (Warsaw, 1986) Banach Center Publ. 23 (PWN, Warsaw, 1989) pp. 339-345.

[7] A. E. Eremenko and M. Yu. Lyubich, 'Dynamical properties of some classes of entire functions', Ann. Inst. Fourier 42 (1992), 989-1020.

[8] M. Jankowski, 'Newton's method for solutions of quasi-Bessel differential equations', Ann. Acad. Sci. Fenn. Ser A.l. Math. 22 (1997), 187-204.

[9] M. Yu. Lyubich, 'The dynamics of rational transforms: the topological picture', Russian Math. Surveys 41 (1986), 43-117.

[10] - 'An analysis of the stability of the dynamics of rational functions', Selecta Math. Soviet. 9 (1990), 69-90.

[11] R. Perez Marco, 'Sur une question de Dulac et Fatou', C. R. Acad. Sci., Paris, Ser. I 321 (1995), 1045-1048.

[12] C. McMullen, 'Area and Hausdorff dimension of Julia sets of entire functions', Trans. Amer. Math. Soc. 300 (1987), 329-342.

[13] M. Misiurewicz, 'On iterates of $e^{27}$, Ergodic Theory Dynam. Systems 1 (1981), 103-106.

[14] P. J. Rippon and G. M. Stallard, 'Iteration of a class of hyperbolic meromorphic functions', Proc. Amer. Math. Soc. 207 (1999), 3251-3258.

[15] G. M. Stallard, 'Entire functions with Julia sets of zero measure', Math. Proc. Cambridge Philos. Soc. 108 (1990), 551-557.

[16] N. Steinmetz, Rational iteration (Walter de Gruyter, Berlin, 1993).

[17] J. H. Zheng, 'Singularities and limit functions in iteration of meromorphic functions', preprint, Tsinghua University.

[18] - 'Singularities and wandering domains in iteration of meromorphic functions', Illinois $J$. Math. 44 (2000), 520-530.

[19] _ - 'Uniformly perfect sets and distortion of holomorphic functions', Nagoya Math. J., to appear.

Department of Mathematical Sciences

Tsinghua University

Beijing 100084

P. R. China

e-mail: jzheng@math.tsinghua.edu.cn 
